\title{
IMPLICACIONES DE LA CLASIFICACIÓN DEL EQUILIBRIO DINÁMICO EN JUGADORAS DE FÚTBOL PARA VARIABLES FUNCIONALES. ESTUDIO CLÍNICO DE INTERVENCIÓN.
}

\author{
Elena Martínez-Martínez, MSc ${ }^{1-2}$; Carlos Manrique-Ortiz, MSc ${ }^{1-2}$; Daniel García-Muñoz, PhD ${ }^{1-2}$. \\ 1. Departamento de Fisioterapia, Centro Superior de Estudios Universitarios La Salle, Universidad Autónoma de Madrid, Madrid, España. \\ 2. Grupo de Investigación Motion in Brains, Instituto de Neurociencia y Ciencias del Movimiento (INCIMOV),
}

\section{Correspondencia:}

Daniel García-Muñoz, PT, PhD. Facultad de Ciencias de la Salud. CESU La Salle. Universidad Autónoma of Madrid. Calle La Salle, $n^{\circ} 10,28023$ Madrid, España Teléfono: (+34) 917401980 Fax: (+34) 913571730 E-Mail: daniel.munoz@lasallecampus.es

\section{Conflicto de Intereses:}

Los autores del presente manuscrito declaran no presentar ningún conflicto de interés y además el presente estudio no ha sido presentado en ningún evento científico.

\section{Financiación}

Los autores declaran no haber recibido financiación/compensación para el desarrollo de esta investigación.

Comité de Ética:

CSEULS-PI-158/2017.

DOI:

https://doi.org/10.37382/jomts.v1i1.10

\section{Recepción del Manuscrito:}

21-Octubre-2017

\section{Aceptación del Manuscrito:}

16- Marzo-2018

Licensed under:

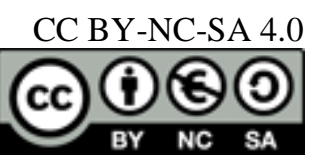

\section{RESUMEN}

Objetivo: Comparar los efectos de un protocolo de fútbol simulado con el SAFT 90, en jugadoras de fútbol femenino con equilibrio dinámico y con desequilibrio dinámico en miembro inferior.

Diseño: Ensayo clínico no controlado.

Marco: Centros participantes: Universidad La Salle Madrid y Equipo Madrid Club de Fútbol Femenino.

Participantes: Los criterios de inclusión fueron: mujeres que practicaran fútbol amateur al menos 2 veces a la semana, asintomáticas y ausencia de lesiones deportivas en los últimos 6 meses. 21 jugadoras de fútbol femenino fueron divididas en dos grupos, (10 jugadoras sin equilibrio dinámico y 11 jugadoras con equilibrio dinámico).

Intervenciones: El desarrollo, la medición y ejecución de las pruebas de la investigación se realizaron en un solo día. Las participantes fueron valoradas por las distintas pruebas antes de la realización del protocolo SAFT 90 y tras la ejecución del protocolo se repitieron las mismas pruebas de valoración. Estas pruebas fueron: El Y Balance Test, El test del salto con caída previa (SCCP), el test de Salto con contramovimiento (SCC) y dinamometría de la musculatura isquiotibial.

Variables: Rigidez de los Tejidos Pasivos (RTP), Índice de Fuerza Reactiva (IFR), tiempo de contacto, velocidad, tiempo de vuelo, fuerza explosiva, potencia y fuerza isométrica.

Resultados: Los resultados mostraron un aumento de la $\mathrm{RTP}(\mathrm{P}=0,03)$, aumento del IFR $(P=0,03)$, disminución de la velocidad $(P=0,04)$ y disminución en el tiempo de Contacto $(\mathrm{P}=0,02)$ en el grupo de las jugadoras sin equilibrio dinámico frente al grupo de jugadoras con equilibrio dinámico.

Conclusión: Las jugadoras de fútbol femenino con desequilibrio dinámico de miembros inferiores tienen diferencias después de una intervención de fútbol simulado, asociándose con mayor riesgo de lesión.

Palabras Clave: Lesiones de fútbol femenino; Y Balance Test; SAFT 90; Rigidez; Fuerza Reactiva. 


\section{INTRODUCCIÓN}

El fútbol es el deporte más popular del mundo, con más de 270 millones de personas que practican este deporte (D’Hooghe, 2016). El fútbol femenino como deporte de equipo está desarrollando un crecimiento ascendente a nivel mundial, la investigación elaborada por la UEFA para la campaña 2016/2017, cuantifica que el número total de jugadoras registradas supera los 1,270 millones. Cada vez son más las jugadoras de fútbol y el número de jugadoras registradas menores de 18 años superan los 827.000. El número de ligas juveniles (entre sub-6 y sub-23) ha crecido de 164 a 266 entre la temporada 2012/13 y 2016/17 (UEFA, 2017).

En España las licencias federativas en fútbol femenino van en aumento. Según los datos de la Real Federación Española de fútbol, se ha pasado de tener 13.582 licencias en la temporada de 2004-2005 a 31.314 licencias en la temporada 2013-2104. Esto significa que hay mayor número de equipos de fútbol femeninos desde los inicios en fútbol base, más competiciones, y más impulso del deporte femenino por parte de las instituciones (Real Federación Española de Fútbol, 2005; Real Federación Española de Fútbol., 2014).

En el estudio de Larruskain et al. (2017) donde se compararon las lesiones en el fútbol femenino con respecto al masculino, se observó mayor incidencia de lesiones graves y mayores ausencias en mujeres, a pesar de tener la mitad de la cantidad de lesiones que los hombres. El número total de días perdidos fue de un $21 \%$ mayor en las mujeres comparado con los hombres (Larruskain et al., 2017) y con respecto al riesgo de ruptura del ligamento cruzado anterior es 23 veces mayor en la mujer que en el hombre (Waldén et al., 2011), debido a las diferencias de sexo en la biomecánica, el control neuromuscular del tronco, la cadera y la rodilla (Hewett et al., 2006).

El fútbol es un deporte con un perfil de actividad irregular e intermitente, donde se desarrollan jugadas de alta intensidad y de gran demanda física, en un partido se produce en un gran número de veces, cambios rápidos de dirección, cambios de ritmo con aceleraciones y desaceleraciones repentinas, saltos y aterrizajes (Faude et al., 2013). Esta demanda física requiere un buen equilibrio. En estudios anteriores se ha utilizado el Y Balance Test (YBT) (Plisky et al., 2006; Dallinga et al., 2012; Gribble et al., 2012), que es una prueba sencilla y barata que evalúa el equilibrio dinámico, es una medida confiable y tiene validez como una prueba dinámica para predecir el riesgo de lesión en las extremidades inferiores, para identificar déficits de equilibrio dinámico en pacientes con lesión en los miembros inferiores y para responder a programas de entrenamiento tanto en personas sanas como personas con lesiones en la extremidad inferior (Plisky et al., 2006; Dallinga et al., 2012; Gribble et al., 2012).

Una aplicación clínica importante del YBT es utilizar el nivel de estabilidad dinámica demostrada en la prueba para predecir el riesgo de lesión en las articulaciones de las extremidades inferiores. Plisky et al. (2006) informaron en el estudio que realizaron con 235 jugadores de baloncesto de escuela secundaria, que los jugadores con diferencias de alcance anteriores de derecha a izquierda de más de $4 \mathrm{~cm}$ eran 2,5 veces más propensos a sufrir lesiones en las extremidades inferiores. $\mathrm{Y}$ que las niñas con una puntuación de alcance compuesto de menos del 94\% de la longitud de su extremidad tenían 6,5 veces más probabilidades de sufrir una lesión en las extremidades inferiores.

Otra investigación ha sugerido que una limitada capacidad de alcance en la dirección posterolateral se asocia a un riesgo elevado de lesiones de tobillo en adultos activos (De Noronha et al., 2013).

Habitualmente en jugadas durante un partido de fútbol que realizan movimientos de alta intensidad $\mathrm{y}$ velocidad como saltos, esprines o cambios de dirección, se requiere una buena actuación de ciclo estiramiento-acortamiento (CEA). Este fenómeno ha sido definido como un pre-activación excéntrica muscular antes del contacto con el suelo seguida de una rápida transición entre la fase excéntrica y la concéntrica (Komi, 2000). Para evaluar la capacidad del CEA, se puede utilizar la medida de la Rigidez de 
los Tejidos Pasivos, (RTP) conocida como "rigidez de pierna” y el Índice de Fuerza Reactiva (IFR).

La RTP se define como la resistencia del cuerpo a la deformación (Brughelli y Cronin, 2008b) se constituye con un componente pasivo proporcionado por huesos, ligamentos y tendones, y un componente muscular activo (Arampatzis et al., 2001). Considerando una sola articulación, la RTP se calcula como el momento de la articulación relativa a su desplazamiento angular (Brughelli y Cronin, 2008a), mientras que la RTP de las extremidades inferiores, se calcula como la fuerza de reacción vertical máxima del suelo con respecto al desplazamiento del centro de masas del cuerpo (Dalleau et al., 2004).

El IFR se define como la relación entre la altura de salto y el tiempo de contacto con el suelo (McClymont, 2003), se utiliza para evaluar la función y el rendimiento en actividades que conlleven un CEA de un atleta, evaluar las capacidades de rebote del atleta, cuantificar el entrenamiento pliométrico y tiene capacidad para controlar la fatiga neuromuscular (Beattie y Flanagan, 2015; Healy et al., 2016).

Hasta la fecha, no hay estudios que relacionen la RTP, el IFR, la velocidad, la fuerza explosiva, la potencia y la fuerza isométrica en fútbol femenino. Teniendo en cuenta la relación de los distintos parámetros involucrados en el rendimiento deportivo y la relevancia del control neuromuscular y del equilibrio dinámico en la disminución del riesgo de lesiones (Myer et al., 2013), el objetivo de este estudio fue comparar los efectos de un protocolo de fútbol simulado con el SAFT 90, en jugadoras de fútbol femenino con equilibrio dinámico y jugadoras con desequilibrio dinámico en las variables funcionales de la RTP, el IFR, la velocidad, la fuerza explosiva, la potencia y la fuerza isométrica.

\section{MÉTODOS}

\section{Participantes}

Un total de 21 mujeres pertenecientes al equipo de Madrid Club de Fútbol Femenino fueron reclutadas para formar parte de este estudio. Los criterios de inclusión fueron: mujeres que practicaran fútbol amateur al menos 2 veces a la semana, asintomáticas y ausencia de lesiones deportivas en los últimos 6 meses.

Se excluyeron del estudio las jugadoras con historia de problemas ortopédicos conocidos, tales como episodios de lesión en músculos isquiotibiales, lesiones en rodilla, fracturas, cirugía o dolor de espalda durante los últimos seis meses previos a la investigación o presencia de DOMS en el día del estudio. Las participantes fueron instruidas para no realizar actividad física vigorosa en las 24 horas previas a la realización del estudio (De Ste Croix et al., 2016). Antes de iniciar la investigación, las participantes fueron informadas de las características del estudio y firmaron un consentimiento informado aprobado por el Comité de ética (CSEULS-PI158/2017) de la Universidad de La Salle Madrid.

\section{Diseño}

El desarrollo, la medición y ejecución de las pruebas de la investigación se realizaron en un solo día. Las participantes fueron valoradas por las distintas pruebas antes de la realización del protocolo SAFT 90 y tras la ejecución del protocolo se repitieron las mismas pruebas de valoración.

\section{Pruebas de valoración}

\section{- Y balance test}

El YBT consiste en una plataforma o base de la que parten tres tubos, el tubo anterior se coloca en un ángulo de 135 grados con respecto a los tubos posteriores, y entre estos habrá una angulación de 45 grados. Cada tubo está marcado en incrementos de 5 centímetros para realizar las medidas La prueba se ejecutó sin calzado (Smith et al., 2015) y con las manos posicionadas en la cadera (Butowicz et al., 2016). La participante se colocó de pie sobre una sola pierna en el centro de la base y se le pidió alcanzar con la extremidad libre la mayor distancia posible en el siguiente orden: dirección anterior, posteromedial y posterolateral con un indicador de alcance (Plisky et al., 2009). 
Cada participante pudo realizar 6 ensayos con cada pierna antes de realizar la prueba. A continuación, se realizaron tres pruebas en la dirección anterior apoyada sobre el pie derecho y tres pruebas en dirección anterior sobre el pie izquierdo. El mismo orden se desarrolló para la dirección posteromedial y la dirección posterolateral. La prueba fue calificada como la suma del mejor esfuerzo de tres ensayos exitosos en cada dirección. La prueba no se consideró valida si la participante no regresó a la postura inicial, si pateó el indicador de alcance en lugar de empujarlo, si se apoyó en el indicador para mantener la postura o no regresó el pie de alcance a la posición inicial bajo control (Shaffer et al., 2013; Hammami et al., 2016).

Se ha reportado una excelente fiabilidad intraevaluador e interevaluador para el YBT con valores ICC que oscilan entre 0,85 y 0,91 intraevaluadores y 0,99 y 1,00 interevaluadores (Čoh y Mackala, 2013; Chimera y Warren, 2016).

Para expresar la distancia de alcance como un porcentaje de la longitud del miembro, el valor normalizado se calcula como la distancia de alcance, que es la suma de las tres direcciones de alcance, dividido por tres veces la longitud del miembro, multiplicado por 100 (Figura 1).

Figura 1. Valor normalizado de la distancia de alcance

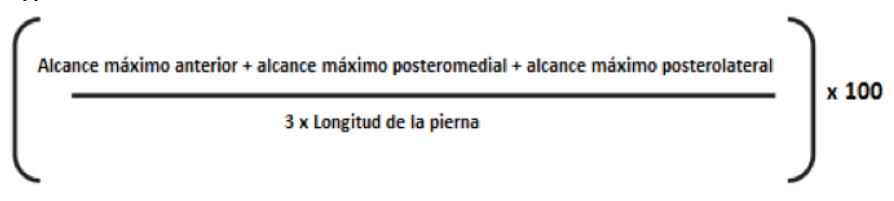

\section{- Test del Salto Con Caída Previa.}

El salto con caída previa, es un movimiento complejo multiarticular, donde la coordinación intermuscular particularmente de agonistas y sinergistas es de gran importancia. El protocolo utilizado en este test es el desarrollado por Bosco que tiene una fiabilidad y una especificidad del 0,93 y 0,97 respectivamente (Čoh y Mackala, 2013).
El participante se posicionó encima de un cajón de $30 \mathrm{~cm}$ de altura (Nilstad et al., 2015), avanzando un pie se dejó caer por el efecto gravitatorio al suelo, recepcionando con ambos pies y con rodillas y caderas flexionadas. Al entrar en contacto con el suelo, el sujeto tenía que realizar inmediatamente un salto vertical máximo (Malloy et al., 2015; Redler et al., 2016). El test se realizó con las manos en la cintura y con zapatillas deportivas. La prueba se grabó y se midió con la aplicación MyJump 2 (BalsalobreFernández et al., 2015) para iPhone. Para medir el salto el investigador se colocó con el teléfono móvil a un metro aproximadamente del deportista, una vez grabado, se marcó el primer fotograma en el cual, los pies dejan de tener contacto con el suelo y el primer fotograma en el que los pies vuelven de nuevo a tocar el suelo, teniendo así el tiempo de vuelo de nuestro deportista, dato que la aplicación usó para calcular la altura de salto, el IFR, el tiempo de contacto y la RTP.

\section{- Salto con contramovimiento}

El test salto con contramovimiento (SCC), ha sido utilizado en numerosos estudios (Shalfawi et al., 2011; Balsalobre-Fernández et al., 2014; RodríguezRosell et al., 2017), para investigar las características físicas de jugadores de fútbol y baloncesto.

En la ejecución del SCC, la participante comenzó desde la posición de bipedestación, seguidamente adoptó una posición de semi-sentadilla con una angulación de rodillas de aproximadamente $90^{\circ}$ (éste es el contramovimiento) y seguidamente ejecutó un despegue del suelo realizando un salto vertical inmediatamente, con la espalda lo más recta posible. Los saltos se realizaron sin movimiento de brazos, los cuales se colocaron en la cadera.

Los parámetros analizados fueron: el perfil de la fuerza, el tiempo de vuelo, la velocidad y la potencia de los saltos. La grabación y medición de los saltos se realizó con la aplicación Myjump 2 (BalsalobreFernández et al., 2015) para iPhone que utiliza la cámara de alta velocidad para grabar vídeos en cámara lenta. 


\section{- Dinamometría de musculatura isquiotibial.}

La fuerza general del miembro inferior y la zona lumbopélvica se midió con un dinamómetro, se utilizó el modelo T.K.K. 5002 de la casa Takei Scientific Instruments. La variable de medida fue el Kilogramo fuerza $(\mathrm{Kg})$. Para la medición se ajustó la barra de manos para que la participante se colocara encima de la plataforma con las rodillas flexionadas a $120^{\circ}$ y la espalda recta. Una vez colocada la participante debía hacer una extensión isométrica de las piernas lo más fuerte posible manteniendo la posición, sin extender la espalda. Se realizaron 3 intentos con un descanso de un minuto entre cada intento y se anotó la medida mayor de las tres (Segovia et al., 2008).

\section{- Protocolo Soccer-specific Aerobic Field Test (SAFT 90)}

El protocolo Soccer-specific Aerobic Field Test (SAFT) está basado en los movimientos obtenidos en 2007 de la competición inglesa de fútbol (con la tecnología Prozone ${ }^{\odot}$ ). Ha sido validado, para replicar la fatiga en un partido de fútbol real (Lovell \& Knaper, 2008). Incluye movimientos multidireccionales y movimientos frecuentes de un partido, además de aceleraciones y desaceleraciones. En el recorrido se colocaran dos conos en paralelo a una distancia total de 20 metros. Dentro de esta distancia se ubicarán cuatro conos a una distancia predeterminada (Figura 2). En el primer cono se realizarán movimientos alternativos prefijados, en los tres restantes se realizará un movimiento de zig- zag, llegando hasta el final y volviendo. La prueba tiene
1269 cambios de velocidad y 1350 cambios de dirección en los 90 minutos que dura el test (Lovell \& Knaper, 2008; Small et al., 2010).

\section{Procedimiento}

Antes de comenzar, se les realizó una serie de valoraciones antropométricas: peso, altura, índice cintura-cadera, pliegues cutáneos y longitud de la pierna, medida por la distancia en centímetros desde la espina iliaca anterosuperior hasta la porción más distal del maléolo medial del tobillo (Plisky et al., 2009).

Se realizó un calentamiento de diez minutos de duración (Jones et al., 2015) que consistió en cinco minutos de trote suave y cinco minutos de ejercicios dinámicos de miembros inferiores tales como: flexión y extensión de caderas, abducción y aducción de caderas, desplazamientos laterales, "skipping" y saltos verticales en el sitio.

Tras el calentamiento se procedió a realizar las pruebas de valoración: YBT, el Test del Salto Con Caída Previa (SCCP), el test de Salto Con Contramovimiento (SCC) y una dinamometría de la musculatura isquiotibial.

Con los resultados de la valoración del YBT se procedió a segmentar la muestra en dos grupos en función del riesgo de lesión (Calvo et al., 2015). El grupo de Equilibrio Dinámico (ED) fue aquel cuya diferencia de distancia de alcance entre la pierna derecha y la izquierda fue menor a $4 \mathrm{~cm}$ y por tanto

Figura 2. Protocolo Soccer-specific Aerobic Field Test
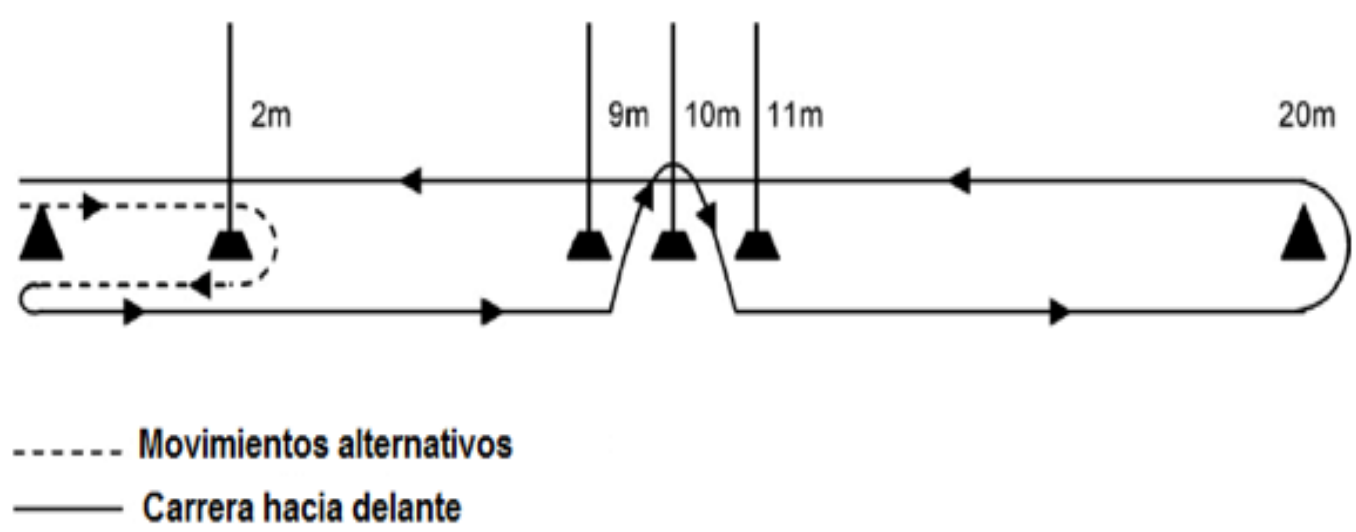
sin riesgo de lesión. El grupo de Desequilibrio Dinámico (DD) lo constituyeron las jugadoras cuya diferencia de distancia de alcance entre la pierna derecha y la izquierda fue igual o mayor a $4 \mathrm{~cm}$ y por tanto con riesgo de lesión.

\section{Análisis estadístico}

Un tercer investigador involucrado en este estudio se encargó del análisis estadístico de forma cegada, donde se compararon los resultados de las variables medidas entre los dos grupos de participantes.

Las variables se comportaban como distribución no paramétrica según la prueba de normalidad de Shapiro-Wilk $(\mathrm{P}<0,01)$. Por ello se utilizó el test estadístico de Wilconxon para evaluar las diferencias entre las variables de muestras relacionadas y la $\mathrm{U}$ de Mann-Whitney para analizar las diferencias entre las variables independientes. Todos los análisis de datos se realizaron en SPSS para Windows, Versión 21.0 (SPSS Inc., Chicago, IL). Los análisis estadísticos se realizaron con un nivel de confianza del $95 \%$ y un valor de $\mathrm{P}$ inferior a 0,05 se consideró estadísticamente significativo.
Figura 3. Diagrama de Flujo.

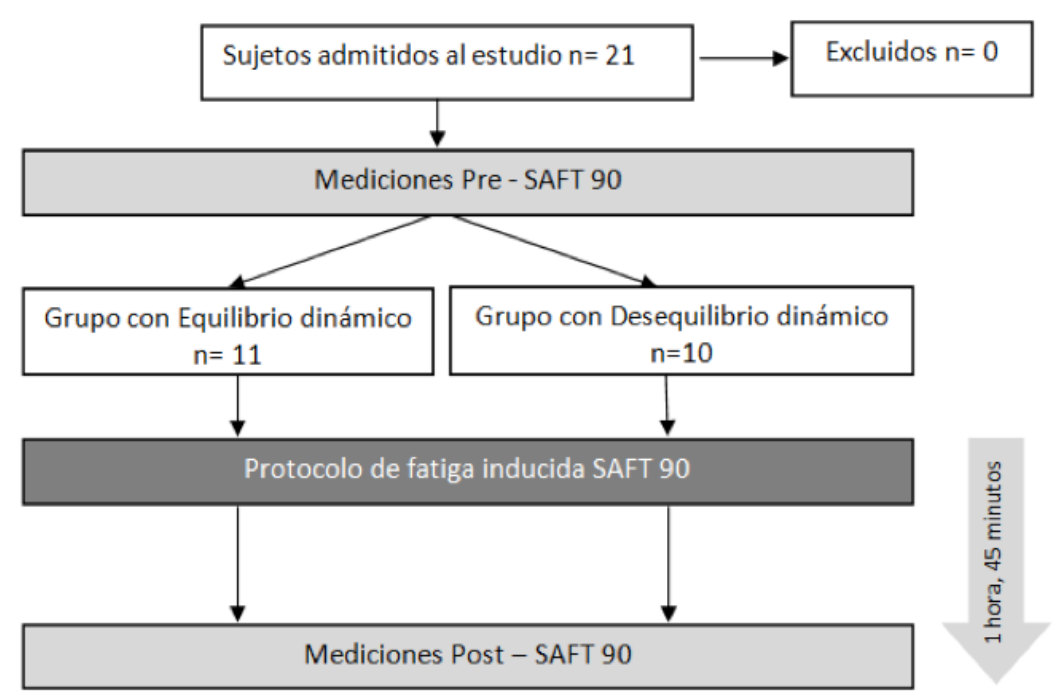

\section{RESULTADOS}

Todas las participantes incluidas en la muestra cumplieron con los criterios de selección y no hubo exclusiones ni pérdidas durante el proceso del ensayo clínico (Figura 3).

De los participantes, 11 pertenecían al grupo ED y 10 pertenecían al grupo DD. Estos dos grupos de pacientes no presentaron diferencias significativas para ninguna de las variables estudiadas. $(\mathrm{P}>0,05)$ (Tabla 1 y Tabla 2).

Tabla 1. Características demográficas de las participantes.

\begin{tabular}{cccccc} 
& \multicolumn{2}{c}{ Grupo ED $n=11$} & \multicolumn{2}{c}{ Grupo DD $n=10$} \\
& Mediana & Rangos & Mediana & Rangos & U Mann-Whitney \\
Edad (años) & 17,00 & $(15,00-19,00)$ & 15,50 & $(15,00-18,00)$ & 0,32 \\
IMC (Kg/m²) & 21,29 & $(19,47-22,03)$ & 21,19 & $(20,19-22,24)$ & 0,75 \\
\% Grasa & 16,94 & $(16,06-21,03)$ & 21,13 & $(18,20-23,50)$ & 0,10 \\
ICC (cm) & 0,76 & $(0,72-0,78)$ & 0,78 & $(0,72-0,81)$ & 0,75 \\
FI (kg) & 80,00 & $(71,00-85,00)$ & 82,50 & $(70-89,50)$ & 0,78
\end{tabular}

ED= Equilibrio Dinámico; DD= Desequilibrio Dinámico; IMC=Índice de Masa Corporal; Índice Cintura Cadera; FI= Fuerza Isométrica 
Tabla 2. Comparativa de las medianas y percentiles entre grupos de los parámetros Pre-SAFT 90.

\begin{tabular}{|c|c|c|c|c|}
\hline & IFR & Tiempo Contacto & RTP & Tiempo Vuelo \\
\hline ED & $0,90(0,82-1,62)$ & $479,17(329,17-583,33)$ & $3,67(2,82-6,57)$ & $470,83(437,50-495,83)$ \\
\hline DD & $0,90(0,80-1,02)$ & $506,25(468,75-607,60)$ & $3,60(2,66-4,52)$ & $479,37(466,67-504,17)$ \\
\hline U Mann-Whitney & 0,53 & 0,24 & 0,40 & 0,34 \\
\hline & Velocidad & Fuerza Explosiva & Potencia & Fuerza Isométrica \\
\hline ED & $1,15(1,07-1,22)$ & $937,60(867,86-1027,55)$ & $1128,02(825,98-1283,85)$ & $80,00(71,00-85,00)$ \\
\hline DD & $1,18(1,14-1,24)$ & $1018,93(861,85-1058,63)$ & $1223,84(988,09-1335,85)$ & $82,50(70,00-89,50)$ \\
\hline U Mann-Whitney & 0,36 & 0,57 & 0,40 & 0,77 \\
\hline
\end{tabular}

$\mathrm{ED}=$ Equilibrio Dinámico DD= Desequilibrio dinámico.

Comparando los resultados entre las variables medidas antes y después de la intervención se encontraron diferencias estadísticamente significativas en el grupo de DD para la variable IFR $(\mathrm{P}=0,03)$,
Tiempo de Contacto $(\mathrm{P}=0,02)$, Rigidez de Tejidos Pasivos $(\mathrm{P}=0,03)$ y Velocidad $(\mathrm{P}=0,04)$. Para ver los resultados de las demás variables véase la Tabla 3 .

Tabla 3. Datos de las medianas y percentiles de los parámetros Pre-SAFT 90 y Post-SAFT 90.

\begin{tabular}{|c|c|c|c|c|}
\hline Variable & Grupos & Pre & Post & $\begin{array}{l}\text { Wilconxon (P- } \\
\text { valor) }\end{array}$ \\
\hline \multirow{2}{*}{ IFR } & ED & $0,90(0,82-1,62)$ & $0,96(0,77-1,31)$ & 0,77 \\
\hline & DD & $0,90(0,80-1,02)$ & $0,96(0,85-1,18)$ & $0,03 *$ \\
\hline \multirow[b]{2}{*}{ Tiempo de Contacto } & ED & $479,17(329,17-583,33)$ & $446(321-575)$ & 1 \\
\hline & DD & $506,25(468,75-607,60)$ & $483,33(440,72-512,50)$ & $0,02 *$ \\
\hline \multirow{2}{*}{ Rigidez Tejidos Pasivos } & ED & $3,67(2,82-6,57)$ & $3,98(2,81-7,27)$ & 0,72 \\
\hline & DD & $3,60(2,66-4,5)$ & $4,27(4,01-4,53)$ & $0,03^{*}$ \\
\hline \multirow{2}{*}{ Tiempo de Vuelo } & ED & $470,83(437,50-495,83)$ & $446,25(416,67-479,17)$ & 0,21 \\
\hline & DD & $479,37(466,67-504,17)$ & $462,50(435,83-487,81)$ & 0,09 \\
\hline \multirow{2}{*}{ Velocidad } & ED & $1,15(1,07-1,22)$ & $1,14(1,07-1,21)$ & 0,61 \\
\hline & DD & $1,18(1,14-1,24)$ & $1,13(1,08-1,16)$ & $0,04 *$ \\
\hline \multirow{3}{*}{ Fuerza Explosiva } & ED & $937,60(867,86-1027,55)$ & $922,29(881,6-996,44)$ & 0,14 \\
\hline & & & & \\
\hline & DD & $1018,93(861,85-1058,63)$ & $938,68(923,49-988,17)$ & 0,44 \\
\hline \multirow{2}{*}{ Potencia } & ED & $1128,00(825,98-1283,85)$ & $1040,39(903,49-1220,19)$ & 0,20 \\
\hline & DD & $1223,84(988,09-1335,85)$ & $1062,46(1000,58-1193,13)$ & 0,14 \\
\hline \multirow{2}{*}{ Fuerza Isométrica } & ED & $80,00(71,00-85,00)$ & $82,00(65,00-89,00)$ & 0,88 \\
\hline & DD & $82,50(70-89,50)$ & $80,50(69,25-85,75)$ & 0,28 \\
\hline
\end{tabular}

$\mathrm{ED}=$ Equilibrio Dinámico , DD= Desequilibrio dinámico; IFR= Índice de Fuerza Reactiva $* \mathrm{P}<0,05$. 
Comparando las diferencias entre grupos post intervención no se encontraron diferencias estadísticamente significativas en las variables (Tabla 4).

\section{DISCUSIÓN}

Este estudio tuvo como principal objetivo comparar los efectos de un protocolo de fútbol simulado con el SAFT 90, en jugadoras de fútbol femenino con equilibrio dinámico y jugadoras con desequilibrio dinámico. Para ello, se analizaron las variables funcionales de la RTP, el IFR, la velocidad, la fuerza explosiva, la potencia y la fuerza isométrica.

El hallazgo principal de este estudio es que sólo las jugadoras con desequilibrio dinámico mostraron cambios en ciertas variables funcionales después de la realización de un partido de fútbol simulado: la RTP, el IFR, la velocidad y el tiempo de contacto.

\section{Rigidez de los Tejidos pasivos}

Los resultados del estudio muestran un aumento de la RTP tras el protocolo SAFT 90 en ambos grupos, siendo esta diferencia estadísticamente significativa sólo para el grupo de desequilibrio dinámico. Estos resultados coinciden con el estudio de Lyle et al. (2014), sin embargo, en esta variable, la literatura es controvertida, encontrándose otros estudios donde se mantiene (Padua et al., 2006; Cone et al., 2012; De Ste Croix et al., 2016) y otro donde disminuye (Lehnert et al., 2016).
El aumento de la RTP en el estudio, podría estar relacionado con mayor riesgo de lesión si tenemos en cuenta los resultados de literatura previa (Butler et al., 2003; Pruyn et al., 2012; Lyle et al., 2014). Butler et al. (2003), sugieren que un nivel de rigidez como adaptación es necesario para un rendimiento óptimo, pero que sin embargo, el aumento de rigidez como respuesta aguda al ejercicio con impacto puede estar relacionado con el aumento del riesgo de lesiones.

El equipo de Lyle et al. (2014) evaluó a 14 jugadoras de fútbol femenino y 15 masculinos de edades entre 15-18 años. Se les realizó un test de destreza de las extremidades inferiores (prueba LED) y los resultados mostraron que las chicas tuvieron menor destreza y una mayor rigidez de las piernas durante una tarea de salto cuando se compararon con los chicos de la misma edad. La mayor rigidez de la pierna observada en las chicas se atribuyó a una mayor fuerza de reacción vertical en el suelo y una disminución del desplazamiento del centro de masas.

Este estudio sugiere que las mujeres desaceleran el impulso del cuerpo mediante la absorción de mayor energía en el tobillo y la rodilla, mientras que los chicos tienden a absorber más energía en la rodilla y de la cadera. Además, se vio que las chicas hacían movimientos más pequeños de la flexión de la rodilla y de la cadera y fuerzas más altas de la reacción de tierra. A este patrón biomecánico se ha identificado como una "estrategia de rigidez".

Tabla 4. Comparativa de las medianas y percentiles entre grupos de los parámetros Post-SAFT 90.

\begin{tabular}{|c|c|c|c|c|}
\hline & IFR & Tiempo Contacto & RTP & Tiempo Vuelo \\
\hline ED & $0,96(0,77-1,31)$ & $446(321-575)$ & $3,98(2,81-7,27)$ & $446,25(416,67-479,17)$ \\
\hline DD & $0,96(0,85-1,18)$ & $483,33(440,72-512,50)$ & $4,27(4,01-4,53)$ & $462,50(435,83-487,81)$ \\
\hline U Mann-Whitney & 0,70 & 0,62 & 0,72 & 0,80 \\
\hline & Velocidad & Fuerza Explosiva & Potencia & Fuerza Isométrica \\
\hline ED & $1,14(1,07-1,21)$ & $922,29(881,6-996,44)$ & $1040,39(903,49-1220,19)$ & $82(65-89)$ \\
\hline DD & $1,13(1,08-1,16)$ & $938,68(923,49-988,17)$ & $1062,46(1000,58-1193,13)$ & $80,50(69,25-85,75)$ \\
\hline U Mann-Whitney & 0,89 & 0,36 & 0,62 & 0,78 \\
\hline
\end{tabular}

Abreviaturas: $\mathrm{ED}=$ Equilibrio Dinámico $\mathrm{DD}=$ Desequilibrio dinámico 
En los estudios de De Ste Croix et al. (2016), Cone et al. (2012), y Padua et al. (2006) se mantuvo la RTP. De Ste Croix et al. (2016), evaluaron la RTP en 37 jugadoras dividiéndolas en tres grupos por edades. Se les midió la RTP antes y después de la realización del protocolo SAFT 90 y los resultados mostraron un aumento significativo de la RTP en las chicas entre 15 y 17 años y un mantenimiento de la rigidez en las chicas de entre 13 y 15 años tras la realización del protocolo.

Cone et al. (2012), evaluaron a 24 jugadores de fútbol de entre 18 y 21 años durante la simulación del partido de fútbol. Los autores de este estudio atribuyeron el mantenimiento de la rigidez a cambios coordinativos que diferían entre los miembros dominantes y no dominantes en relación a la fuerza vertical de reacción del suelo y el desplazamiento de masas.

En el estudio de Padua et al. (2006), se evaluó la influencia de la fatiga inducida a través de ejercicio submáximo prolongado en cadena cinética cerrada en hombres y mujeres. Los resultados mostraron un mantenimiento de la rigidez vertical y el desarrollo de la activación muscular y estrategias de movimiento articular para satisfacer las demandas físicas de la tarea realizada.

El estudio que mostró una disminución de la RTP fue el de Lehnert et al. (2016). En este estudio se evaluaron los efectos en el control muscular y neuromuscular tras el protocolo SAFT 90 en 18 jugadores de entre 14 y 15 años.

Los resultados en la literatura previa son variados y pueden estar influenciados por la edad de maduración de los participantes. Aunque nuestra muestra coincide con las mismas edades de estos estudios, no podemos comparar los resultados ya que en nuestro estudio se hizo una clasificación en función del equilibrio dinámico. Los resultados de este estudio pueden tener implicaciones para clínicos y futuros investigadores que quieran clasificar a las mujeres futbolistas según su equilibrio dinámico para posteriormente cuantificar las variables funcionales que se han relacionado anteriormente con riesgo de lesión.

\section{Índice de Fuerza Reactiva}

La literatura actual identifica esta variable como un predictor, entre otros, de lesión del ligamento cruzado anterior. Se cree que un nivel bajo de este índice es indicativo de una pobre capacidad del CEA, probablemente debido a una mayor latencia muscular y por tanto una baja actividad neuromuscular (Beattie \& Flanagan, 2015).

Tras el protocolo SAFT 90, el grupo DD mostró un aumento estadísticamente significativo en el IFR y una disminución significativa en el tiempo de contacto. Estos resultados difieren con estudios más recientes que evalúan el IFR en deportistas (Lehnert et al., 2016; Wilke et al., 2016). En el estudio de Lehnert et al. (2016), en 18 jugadores de fútbol con una media de 14,4 años, obtuvieron niveles significativamente más bajos en el IFR tras la realización del protocolo SAFT 90. Y en el otro estudio llevado a cabo por el equipo de Wilke et al. (2016), los resultados mostraron un menor IFR tras la realización de un protocolo de agilidad funcional (FAST-FP: functional agility short-term fatigue protocol) donde se realizaban cargas y habilidades características de los deportes de equipo como esprines, cambios de dirección, saltos y sentadillas.

Los diferentes resultados encontrados en el presente estudio podrían deberse a que nuestro estudio hizo una segmentación de la muestra, clasificando a las jugadoras en función de su equilibrio dinámico. Únicamente las jugadoras con desequilibrio dinámico mostraron un aumento significativo del IFR a diferencia de los estudios mencionados anteriormente.

\section{Fuerza Explosiva, Velocidad y Potencia}

Las variables medidas a través del test del salto con contramovimiento, fuerza explosiva, velocidad y potencia, muestran descensos para ambos grupos, pero siendo solo estadísticamente significativo el descenso de la velocidad para el grupo sin equilibrio dinámico. 
Pérdidas de velocidad en distintos tipos de ejercicio ya se han reportado en diferentes estudios (Andersson et al., 2008; Jiménez-Reyes et al., 2011; Sánchez-Medina \& González-Badillo, 2011; Balsalobre-Fernández et al., 2014) y muestran que menor velocidad en el SCC está relacionada con fatiga. Estos resultados van en la misma línea que los encontrados en este artículo donde sólo las mujeres sin ED mostraron un descenso en la velocidad.

En el estudio de Sánchez-Medina \& GonzálezBadillo (2011) cuyo objetivo fue analizar la respuesta aguda mecánica y metabólica a protocolos de ejercicio de resistencia, se encontró disminución de la velocidad y pérdidas de altura del SCC tras la realización de los protocolos.

Jiménez-Reyes et al. (2011) asociaron la pérdida de velocidad con la reducción de la fuerza. Balsalobre et al. (2014), analizaron el desempeño del SCC después de una competición de corredores de media y larga distancia y también mostraron una disminución de las capacidades del SCC.

Anderson et al. (2008) valoraron los cambios en los parámetros neuromusculares y bioquímicos en respuesta a un partido de fútbol femenino seguido de $72 \mathrm{~h}$ de recuperación y de un segundo partido. Los hallazgos tras el primer partido fueron una reducción significativa en la velocidad del sprint, del SCC y de la fuerza isocinética.

Aunque los resultados de nuestro estudio se asemejan a los de la literatura previa no se pueden comparar debido al diferente procedimiento. Los resultados de este estudio pueden tener implicaciones para clínicos y futuros investigadores que quieran clasificar a las mujeres futbolistas según su equilibrio dinámico para posteriormente cuantificar el descenso de la velocidad que se ha relacionado con riesgo de lesión.

\section{Fuerza isométrica}

El grupo sin equilibrio dinámico mostró una tendencia con menores valores de fuerza isométrica tras realizar el protocolo de intervención, pero no fueron estadísticamente significativos. Sin embargo, literatura previa si ha encontrado un descenso de la fuerza isométrica tras un esfuerzo prolongado (Robineau et al., 2012; Marshall et al., 2014).

El grupo de Robineau et al. (2012) encontraron resultados significativos de descensos de fuerza con sólo 45 minutos de tiempo simulado. Esto puede ser explicado por la repetición de los esfuerzos de tipo explosivos en las acciones específicas del fútbol, estando los músculos extensores de rodilla más predispuestos a la fatiga en comparación a los flexores, debido a su mayor contribución.

Estos datos son similares a los descritos por Marshall et al. (2014), que estudiaron los cambios en la fuerza, en la misma intervención de fútbol simulado. Los resultados mostraron que la fuerza se redujo alrededor de un $8 \%$ a los 45 minutos, así como la velocidad y que se relacionan directamente con el riesgo de lesiones.

Nuestros resultados son similares a la literatura previa, sin embargo, no se pueden comparar porque en nuestro estudio se hizo una clasificación en función del equilibrio dinámico. Futuros estudios deberían ampliar el tamaño muestral para confirmar estos resultados clasificando a las mujeres según su equilibrio dinámico para posteriormente cuantificar la disminución de la fuerza isométrica que anteriormente se ha relacionado con riesgo de lesión.

\section{Limitaciones}

Los resultados del presente estudio deben ser tratados con precaución porque el cálculo del tamaño muestral no fue calculado, sin embargo, estudios similares ya publicados tienen tamaños muestrales similares (De Ste Croix et al., 2016; Lehnert et al., 2016). Otra limitación es que no se realizó la prueba del SCCP de manera monopodal ya descrito en la literatura (Taylor et al., 2016; Tamura et al., 2017), donde se hubiera podido relacionar la fuerza dinámica de cada pierna. Para futuras investigaciones esta valoración puede mostrar datos interesantes en aspectos funcionales del rendimiento. Otra limitación es que no se tuvo en cuenta la fase ovulatoria de las 
participantes en relación a la presencia o no de hiperlaxitud (Shultz et al., 2005; Hicks-Little et al., 2007; Park et al., 2009).

\section{CONCLUSIÓN}

Las mujeres con desequilibrio dinámico en los miembros inferiores mostraron diferencias significativas en variables como la RTP, el IFR, la velocidad y el tiempo de contacto. Esta interpretación debería ser tomada con cautela por tratarse de un estudio piloto.

\section{REFERENCIAS}

Andersson H, Raastad T, Nilsson J, Paulsen G, Garthe I, Kadi F. Neuromuscular fatigue and recovery in elite female soccer: effects of active recovery. Med Sci Sports Exerc. 2008;40(2):372-80 DOI: http://dx.doi.org/10.1249/mss.0b013e31815b8497.

Arampatzis A, Schade F, Walsh M, Brüggemann GP. Influence of leg stiffness and its effect on myodynamic jumping performance. J Electromyogr Kinesiol. 2001;11(5):355-64.

Balsalobre-Fernández C, Glaister M, Lockey RA. The validity and reliability of an iPhone app for measuring vertical jump performance. J Sports Sci. 2015;33(15):1574-9 DOI: http://dx.doi.org/10.1080/02640414.2014.996184.

Balsalobre-Fernández C, Tejero-González CM, del CampoVecino J, Bavaresco N. The concurrent validity and reliability of a low-cost, high-speed camera-based method for measuring the flight time of vertical jumps. J strength Cond Res. 2014;28(2):528-33 DOI: http://dx.doi.org/10.1519/JSC.0b013e318299a52e.

Beattie K, Flanagan EP. Establishing the reliability and meaningful change of the drop-jump reactive strength index. J Aust Strength Cond. 2015;23(5):12-8.

Brughelli M, Cronin J. A review of research on the mechanical stiffness in running and jumping: methodology and implications. Scand J Med Sci Sports. 2008a;18(4):417-26 DOI: http://dx.doi.org/10.1111/j.1600-0838.2008.00769.x.

Brughelli M, Cronin J. Influence of running velocity on vertical, leg and joint stiffness : modelling and recommendations for future research. Sports Med. 2008b;38(8):647-57.

Butler RJ, Crowell HP, Davis IM. Lower extremity stiffness: implications for performance and injury. Clin Biomech. 2003;18(6):511-7 DOI: http://dx.doi.org/10.1016/S02680033(03)00071-8.

Butowicz CM, Ebaugh DD, Noehren B, Silfies SP. Validation of Two Clinical Measures of Core Stability. Int J Sports Phys Ther. 2016;11(1):15-23.

\section{$>$ FRASES DESTACADAS}

$>$ Las jugadoras de futbol con desequilibrio dinámico en el miembro inferior mostraron diferencias después de la intervención con un partido de futbol simulado (SAFT 90).

$>$ Únicamente el grupo de jugadoras de futbol con desequilibrio dinámico mostró diferencia en la RTP, el IFR y la velocidad de las extremidades inferiores después de la intervención.

$>$ La prueba Y Balance Test puede ser una herramienta de sub-clasificación interesante para clínicos que trabajen con mujeres futbolistas.

Calvo A, Pina JA, Maciá L. Relationship between the Y Balance Test Scores and soft tissue injury incidence in a soccer team. Int J Sports Phys Ther. 2015;10(7):955-66.

Chimera NJ, Warren M. Use of clinical movement screening tests to predict injury in sport. World J Orthop. 2016;7(4):202 DOI: http://dx.doi.org/10.5312/wjo.v7.i4.202.

Čoh M, Mackala K. Differences Between the Elite and Subelite Sprinters in Kinematic and Dynamic Determinations of Countermovement Jump and Drop Jump. J Strength Cond Res. 2013;27(11):3021-7 DOI: http://dx.doi.org/10.1519/JSC.0b013e31828c14d8.

Cone JR, Berry NT, Goldfarb AH, Henson RA, Schmitz RJ, Wideman L, Shultz SJ. Effects of an individualized soccer match simulation on vertical stiffness and impedance. J strength Cond Res. 2012;26(8):2027-36 DOI: http://dx.doi.org/10.1519/JSC.0b013e31823a4076.

D'Hooghe M. Why is UEFA carrying out injury studies? Br J Sports Med. 2016;50(12):707 DOI: http://dx.doi.org/10.1136/bjsports-2016-096334.

Dalleau G, Belli A, Viale F, Lacour JR, Bourdin M. A simple method for field measurements of leg stiffness in hopping. Int J Sports Med. 2004;25(3):170-6 DOI: http://dx.doi.org/10.1055/s-2003-45252.

Dallinga JM, Benjaminse A, Lemmink KAPM. Which screening tools can predict injury to the lower extremities in team sports?: a systematic review. Sports Med. 2012;42(9):791815 DOI: http://dx.doi.org/10.2165/11632730-00000000000000.

Faude O, Rößler R, Junge A. Football injuries in children and adolescent players: are there clues for prevention? Sports Med. 2013;43(9):819-37 DOI: http://dx.doi.org/10.1007/s40279-013-0061-x 
Gribble PA, Hertel J, Plisky P. Using the Star Excursion Balance Test to assess dynamic postural-control deficits and outcomes in lower extremity injury: a literature and systematic review. J Athl Train. 2012;47(3):339-57 DOI: http://dx.doi.org/10.4085/1062-6050-47.3.08.

Hammami R, Granacher U, Makhlouf I, Behm DG, Chaouachi A. Sequencing Effects of Balance and Plyometric Training on Physical Performance in Youth Soccer Athletes. J strength Cond Res. 2016;30(12):3278-89 DOI: http://dx.doi.org/10.1519/JSC.0000000000001425.

Healy R, Kenny IC, Harrison AJ. Section I - Kinesiology Assessing Reactive Strength Measures in Jumping and Hopping Using the Optojump TM System. J Hum Kinet. 2016;54(54):23-32 DOI: http://dx.doi.org/10.1515/hukin2016-0032.

Hewett TE, Myer GD, Ford KR. Anterior Cruciate Ligament Injuries in Female Athletes: Part 1, Mechanisms and Risk Factors. Am J Sports Med. 2006;34(2):299-311 DOI: http://dx.doi.org/10.1177/0363546505284183.

Hicks-Little CA, Thatcher JR, Hauth JM, Goldfuss AJ, Cordova ML. Menstrual cycle stage and oral contraceptive effects on anterior tibial displacement in collegiate female athletes. J Sports Med Phys Fitness. 2007;47(2):255-60.

Jiménez-Reyes P., Cuadrado-Peñafiel V., Gonzalez- Badillo JJ. Application of the Counter Movement Jump Test to Monitor Training Load in Sprint Sessions. Cult Cienc y Deport. 2011;6(17):105-12.

Jones RI, Ryan B, Todd AI. Muscle fatigue induced by a soccer match-play simulation in amateur Black South African players. J Sports Sci. 2015;33(12):1305-11 DOI: http://dx.doi.org/10.1080/02640414.2015.1022572.

Komi P V. Stretch-shortening cycle: a powerful model to study normal and fatigued muscle. J Biomech. 2000;33(10):1197206.

Larruskain J, Lekue JA, Diaz N, Odriozola A, Gil SM. A comparison of injuries in elite male and female football players: A five-season prospective study. Scand J Med Sci Sports. 2017; DOI: http://dx.doi.org/10.1111/sms.12860.

Lehnert M, De Ste Croix M, Zaatar A, Hughes J, Varekova R, Lastovicka O. Muscular and neuromuscular control following soccer-specific exercise in male youth: Changes in injury risk mechanisms. Scand J Med Sci Sports. 2016; DOI: http://dx.doi.org/10.1111/sms.12705.

Lovell R. , Knaper B. SK. Physiological responses to SAFT90: a new soccer-specific match simulation. 2008. p. 46-67.

Lyle MA, Valero-Cuevas FJ, Gregor RJ, Powers CM. Control of dynamic foot-ground interactions in male and female soccer athletes: females exhibit reduced dexterity and higher limb stiffness during landing. J Biomech. 2014;47(2):512-7 DOI: http://dx.doi.org/10.1016/j.jbiomech.2013.10.038.

Malloy P, Morgan A, Meinerz C, Geiser C, Kipp K. The association of dorsiflexion flexibility on knee kinematics and kinetics during a drop vertical jump in healthy female athletes. Knee Surg Sports Traumatol Arthrosc. 2015;23(12):3550-5 DOI: http://dx.doi.org/10.1007/s00167014-3222-z.
Marshall PWM, Lovell R, Jeppesen GK, Andersen K, Siegler JC. Hamstring muscle fatigue and central motor output during a simulated soccer match. PLoS One. 2014;9(7):e102753 DOI: http://dx.doi.org/10.1371/journal.pone.0102753.

McClymont D. Use of the reactive strength index (RSI) as an indicator of plyometric training conditions. Sci Footb V Proc Fifth World Congr Sport Sci Footb. Lisbon, Portugal; 2003.

Myer GD, Sugimoto D, Thomas S, Hewett TE. The influence of age on the effectiveness of neuromuscular training to reduce anterior cruciate ligament injury in female athletes: a metaanalysis. Am J Sports Med. 2013;41(1):203-15 DOI: http://dx.doi.org/10.1177/0363546512460637.

Nilstad A, Krosshaug T, Mok K-M, Bahr R, Andersen TE. Association Between Anatomical Characteristics, Knee Laxity, Muscle Strength, and Peak Knee Valgus During Vertical Drop-Jump Landings. J Orthop Sports Phys Ther. 2015;45(12):998-1005

DOI: http://dx.doi.org/10.2519/jospt.2015.5612.

De Noronha M, França LC, Haupenthal A, Nunes GS. Intrinsic predictive factors for ankle sprain in active university students: a prospective study. Scand J Med Sci Sports. 2013;23(5):541-7 DOI: http://dx.doi.org/10.1111/j.16000838.2011.01434.x.

Padua DA, Arnold BL, Perrin DH, Gansneder BM, Carcia CR, Granata KP. Fatigue, vertical leg stiffness, and stiffness control strategies in males and females. J Athl Train. 2006;41(3):294-304.

Park S-K, Stefanyshyn DJ, Loitz-Ramage B, Hart DA, Ronsky JL. Changing hormone levels during the menstrual cycle affect knee laxity and stiffness in healthy female subjects. Am J Sports Med. 2009;37(3):588-98 DOI: http://dx.doi.org/10.1177/0363546508326713.

Plisky PJ, Gorman PP, Butler RJ, Kiesel KB, Underwood FB, Elkins B. The reliability of an instrumented device for measuring components of the star excursion balance test. $\mathrm{N}$ Am J Sports Phys Ther. 2009;4(2):92-9.

Plisky PJ, Rauh MJ, Kaminski TW, Underwood FB. Star Excursion Balance Test as a predictor of lower extremity injury in high school basketball players. J Orthop Sports Phys Ther. 2006;36(12):911-9 DOI: http://dx.doi.org/10.2519/jospt.2006.2244.

Pruyn EC, Watsford ML, Murphy AJ, Pine MJ, Spurrs RW, Cameron ML, Johnston RJ. Relationship between leg stiffness and lower body injuries in professional Australian football. J Sports Sci. 2012;30(1):71-8 DOI: http://dx.doi.org/10.1080/02640414.2011.624540.

Real Federación Española de Fútbol. Licencias de Fútbol en España en la temporada 2004-2005. 2005;

Real Federación Española de Futbol. Licencias de fútbol en España en la actividad del año 2013-2014. 2014;

Redler LH, Watling JP, Dennis ER, Swart E, Ahmad CS. Reliability of a field-based drop vertical jump screening test for ACL injury risk assessment. Phys Sportsmed. 2016;44(1):46-52 DOI: http://dx.doi.org/10.1080/00913847.2016.1131107. 
Robineau J, Jouaux T, Lacroix M, Babault N. Neuromuscular Fatigue Induced by a 90-Minute Soccer Game Modeling. J Strength Cond Res. 2012;26(2):555-62 DOI: http://dx.doi.org/10.1519/JSC.0b013e318220dda0.

Rodríguez-Rosell D, Mora-Custodio R, Franco-Márquez F, Yáñez-García JM, González-Badillo JJ. Traditional vs. Sport-Specific Vertical Jump Tests: Reliability, Validity, and Relationship With the Legs Strength and Sprint Performance in Adult and Teen Soccer and Basketball Players. J strength Cond Res. 2017;31(1):196-206 DOI: http://dx.doi.org/10.1519/JSC.0000000000001476.

Sánchez-Medina L, González-Badillo JJ. Velocity loss as an indicator of neuromuscular fatigue during resistance training. Med Sci Sports Exerc. 2011;43(9):1725-34 DOI: http://dx.doi.org/10.1249/MSS.0b013e318213f880.

Segovia JC, Lopez-Silvarrey FJ, Legido JC. Manual de valoración funcional: aspectos clínicos y fisiológicos. 2a. Madrid: Elsevier; 2008.

Shaffer SW, Teyhen DS, Lorenson CL, Warren RL, Koreerat CM, Straseske CA, Childs JD. Y-balance test: a reliability study involving multiple raters. Mil Med. 2013;178(11):1264-70 DOI: http://dx.doi.org/10.7205/MILMED-D-13-00222.

Shalfawi SAI, Sabbah A, Kailani G, Tønnessen E, Enoksen E. The relationship between running speed and measures of vertical jump in professional basketball players: a field-test approach. J strength Cond Res. 2011;25(11):3088-92 DOI: http://dx.doi.org/10.1519/JSC.0b013e318212db0e.

Shultz SJ, Sander TC, Kirk SE, Perrin DH. Sex differences in knee joint laxity change across the female menstrual cycle. J Sports Med Phys Fitness. 2005;45(4):594-603.

Small K, McNaughton L, Greig M, Lovell R. The effects of multidirectional soccer-specific fatigue on markers of hamstring injury risk. J Sci Med Sport. 2010;13(1):120-5 DOI: http://dx.doi.org/10.1016/j.jsams.2008.08.005.
Smith CA, Chimera NJ, Warren M. Association of y balance test reach asymmetry and injury in division I athletes. Med Sci Sports Exerc. 2015;47(1):136-41 DOI: http://dx.doi.org/10.1249/MSS.0000000000000380.

De Ste Croix M, Hughes J, Lloyd RS, Oliver JL, Read P. Leg Stiffness In Female Soccer Players. J Strength Cond Res. 2016;1

DOI: http://dx.doi.org/10.1519/JSC.0000000000001715.

Tamura A, Akasaka K, Otsudo T, Shiozawa J, Toda Y, Yamada K. Fatigue influences lower extremity angular velocities during a single-leg drop vertical jump. J Phys Ther. 2017;29:498-504.

Taylor JB, Ford KR, Nguyen A-D, Shultz SJ. Biomechanical Comparison of Single- and Double-Leg Jump Landings in the Sagittal and Frontal Plane. Orthop J Sport Med. SAGE Publications; 2016;4(6):2325967116655158 DOI: http://dx.doi.org/10.1177/2325967116655158.

UEFA. Women's Football across the national associations 20152016. 2017;

Waldén M, Hägglund M, Werner J, Ekstrand J. The epidemiology of anterior cruciate ligament injury in football (soccer): a review of the literature from a gender-related perspective. Knee Surg Sports Traumatol Arthrosc. 2011;19(1):3-10 DOI: http://dx.doi.org/10.1007/s00167-010-1172-7.

Wilke J, Fleckenstein J, Krause F, Vogt L, Banzer W. Sportspecific functional movement can simulate aspects of neuromuscular fatigue occurring in team sports. Sport Biomech. 2016;15(2):151-61 DOI: http://dx.doi.org/10.1080/14763141.2016.1159322. 